BNL - 68093

Informal Report

\title{
OPERATION OF A LOW EMITTANCE LATTICE AT THE NSLS X-RAY RING
}

\author{
E.B. Blum, R. Heese, R. Klaffky, S. Krinsky and J. Safranek \\ National Synchrotron Light Source \\ Brookhaven National Laboratory \\ Upton, NY 11973, USA
}

March 1999

\section{National Synchrotron Light Source}

\author{
Brookhaven National Laboratory \\ Operated by \\ Brookhaven Science Associates \\ Upton, NY 11973
}

Under Contract with the United States Department of Energy

Contract Number DE-AC02-98CH10886 


\section{DISCLAIMER}

This report was prepared as an account of work sponsored by an agency of the United States Government. Neither the United States Government nor any agency thereof, nor any of their employees, nor any of their contractors, subcontractors or their employees, makes any warranty, express or implied, or assumes any legal liability or responsibility for the accuracy, completeness, or any third party's use or the results of such use of any information, apparatus, product, or process disclosed, or represents that its use would not infringe privately owned rights. Reference herein to any specific commercial product, process, or service by trade name, trademark, manufacturer, or otherwise, does not necessarily constitute or imply its endorsement, recommendation, or favoring by the United States Government or any agency thereof or its contractors or subcontractors. The views and opinions of authors expressed herein do not necessarily state or reflect those of the United States Government or any agency thereof or its contractors or subcontractors. The views and opinions of authors expresses herein do not necessarily state to reflect those of the United States Government or any agency thereof. 


\title{
OPERATION OF A LOW EMITTANCE LATTICE AT THE NSLS X-RAY RING*
}

\author{
E.B. Blum ${ }^{\#}$, R. Heese, R. Klaffky, S. Krinsky, BNL, Upton, NY \\ J. Safranek, SSRL, SLAC, Stanford, CA
}

\section{Abstract}

The NSLS X-Ray Ring is now being operated with a low emittance lattice. The horizontal emittance was reduced to $45 \mathrm{~nm}$-rad from $90 \mathrm{~nm}$-rad at $2.584 \mathrm{GeV}$ while maintaining a vertical emittance of $0.1 \mathrm{~nm}$-rad. The electron beam life-time was unaffected by the emittance reduction because the decrease in the dispersion in the dipole magnets compensate for the higher bunch density in the Touschek effect. The lattice will also be implemented at $2.8 \mathrm{GeV}$ after the strength of the focusing sextupoles is increased. The effect of low emittance operation on the synchrotron radiation users will be discussed.

\section{INTRODUCTION}

The NSLS X-Ray Ring has normally operated in a lattice with horizontal and vertical tunes of 9.14 and 6.20, respectively. The horizontal and vertical emittances were $90 \mathrm{~nm}-\mathrm{rad}$ and $0.1 \mathrm{~nm}-\mathrm{rad}$. In the lattice design, the dispersion was constrained to be zero in the long straight sections. By relaxing this constraint, Safranek previously showed ${ }^{[1]}$ that the horizontal emittance could be reduced to as little as $21 \mathrm{~nm}$-rad with the same tunes at the cost of an excessive reduction in the dynamic aperture. He also obtained another lattice with a $38 \mathrm{~nm}$-rad horizontal emittance and a dynamic aperture that was at least as big as the physical aperture everywhere in the ring. Unfortunately, the sextupole strength needed to correct the chromaticity of the $38 \mathrm{~nm}$-rad lattice was greater than could be supplied by the existing sextupole magnets. Even so, a $50 \mathrm{~mA}$ electron beam could be stored before the onset of the head-tail instability.

To obtain a lower emittance without unreasonable increases in the sextupole strengths, a new lattice was developed with horizontal and vertical tunes of 9.83 and 5.71, respectively, producing a horizontal emittance of $45 \mathrm{~nm}$-rad. The vertical emittance has remained unchanged at $0.1 \mathrm{~nm}-\mathrm{rad}$. Operating in this lattice required changes to the injection conditions and to the programs that are followed as the storage ring energy is ramped between the $750 \mathrm{MeV}$ injection energy and the $2.584 \mathrm{GeV}$ operating energy. Currents as high as $500 \mathrm{~mA}$ have been stored using this lattice and $350 \mathrm{~mA}$ currents have been routinely stored for synchrotron radiation operations since September, 1998. The dramatic decrease in the beam size in the low emittance lattice is apparent from the digitized synchrotron radiation profiles in figure 1.
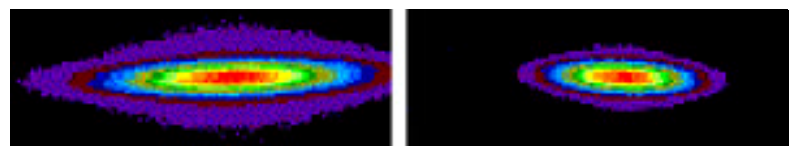

Figure 1: Digitized synchrotron radiation profiles in the original (left) and low emittance (right) lattices. The machine functions at the radiation source points are: (old) $\beta_{x}=1.31 \mathrm{~m}, \beta y=12.2 \mathrm{~m}$; (new) $\beta_{x}=1.04 \mathrm{~m}$, $\beta y=16.2 \mathrm{~m}$.

\section{LATTICE}

The emittance $\varepsilon_{x}$ of a storage ring is ${ }^{[2]}$

\footnotetext{
"Work supported by the U.S Department of Energy under contract DE-AC02-98CH10086

\# Email: blum@bnl.gov
} 


$$
\varepsilon_{x}=\frac{C E^{2}}{\rho} \frac{\int_{D}\left(\beta_{x} \eta_{x}^{\prime 2}+2 \alpha_{x} \eta_{x} \eta_{x}^{\prime}+\gamma_{x} \eta_{x}^{2}\right) d s}{\int_{D} d s},
$$

where $C=1.47 \times 10^{-6} \mathrm{~m} / \mathrm{GeV}^{2}, E$ is the ring energy, $\rho$ is the dipole magnet bending radius, $\beta_{x}$ is the horizontal beta function, $\eta_{x}$ is the horizontal dispersion, $\alpha_{x}=-\beta^{\prime} / 2, \gamma_{x}=\left(1+\alpha_{x}^{2}\right) / \beta_{x}, s$ is the longitudinal position around the ring, and the integrals are taken over all of the dipole magnets. The horizontal beam size at any point in the ring is

$$
\sigma_{x}(s)=\sqrt{\varepsilon_{x} \beta_{x}(s)+\eta_{x}^{2} \sigma_{E}^{2}},
$$

where $\sigma_{E}$ is the energy spread

The NSLS X-Ray Ring was designed with a long straight section in each of the eight super-periods to provide space for injection components, RF cavities, wigglers and undulators for synchrotron radiation production. The original lattice was designed with zero dispersion in the long straight sections to minimize the beam size in the insertion devices by eliminating the second term in eqn. 2 and also to avoid any increase in the emittance from dispersion in the dipole field of a wiggler.

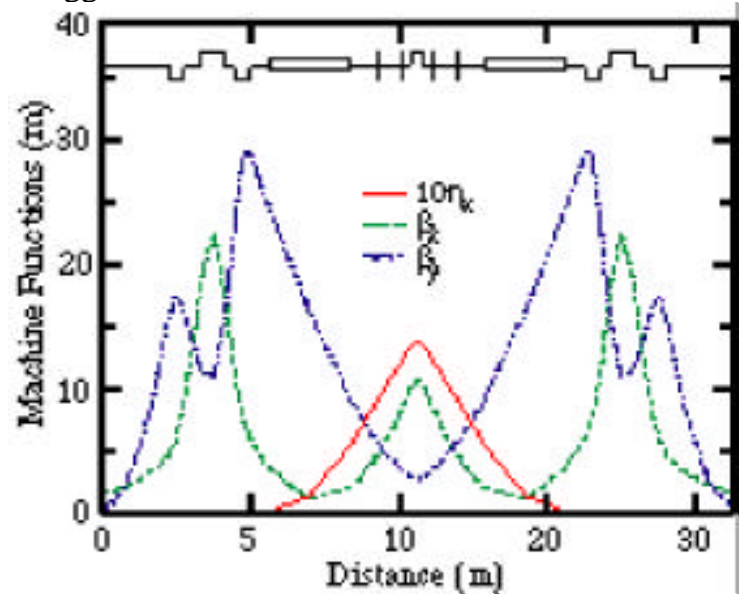

Figure 2. Machine functions in the original lattice.

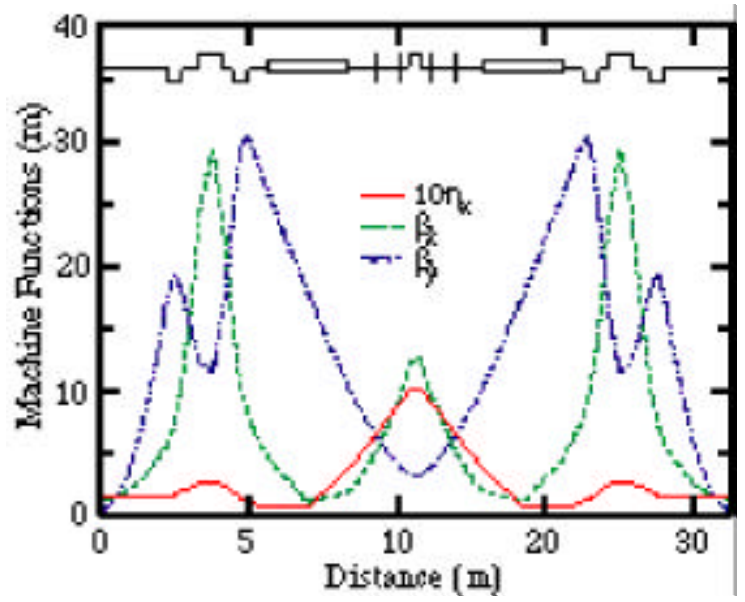

Figure 3. Machine functions in the low emittance lattice.

The properties of the X-Ray Ring lattice can be varied by adjusting the strengths of the four quadrupole families. Permitting non-zero dispersion in the long straight sections was found to reduce the emittance by lowering $\eta_{x}$ and $\eta_{x}^{\prime}$ in the dipole magnets. The reduction in emittance more than compensated for the contribution to the beam size from straight section dispersion. The presence of dispersion in the wigglers located in the long straight sections did increase the emittance somewhat but the effect was small. A calculation shows that the dispersion in the $4.7 \mathrm{~T}, 5$ 
pole superconducting wiggler increases the emittance from 43.3 to $45.6 \mathrm{~nm}-\mathrm{rad}$. Machine functions in the original and low emittance lattices are shown in figures 2 and $3 .$.

To increase the dynamic aperture, a higher emittance lattice with weaker sextupoles but the same tunes as the low emittance lattice is used for injection at $750 \mathrm{MeV}$. When the storage ring is ramped from the injection energy to the $2.584 \mathrm{GeV}$ operating energy, the lattice remains in the injection configuration until the ring energy reaches approximately $2.1 \mathrm{GeV}$. Then, the quadrupole $k$ values are linearly interpolated from the injection values to the low emittance values as the energy is increased from 2.1 to $2.584 \mathrm{GeV}$.

In the past, there has been a significant disagreement between the design and measured optics in the X-Ray Ring

[3]. The discrepancy is mainly caused by the gradient errors produced by an orbit displacement in the sextupoles. The orbit error can not be easily corrected because the synchrotron radiation beamlines have been aligned to the displaced orbit. Instead, trim power supplies were connected to the quadrupole that is adjacent to the sextupoles in each of the eight superperiods. The trim current was determined by using the LOCO program [3] to find the quadrupole strengths that best restored the eight-fold periodicity of the measured orbit response matrix. This restored the machine functions to the design values.

An algorithm, sigycor, has been developed to correct the coupling of the horizontal and vertical betatron motion in the X-Ray Ring ${ }^{[4]}$. The algorithm uses the displacement in the vertical orbit with horizontal steering magnet field as a measure of the vertical coupling. The skew quadrupoles are then adjusted to minimize this coupling. The horizontal and vertical tunes were also varied to find where sigycor was most effective at minimizing the coupling. Following this procedure, a vertical emittance of approximately $0.1 \mathrm{~nm}$-rad was achieved. This is the same as the vertical emittance obtained with the original optics.

\section{ELECTRON BEAM LIFE-TIME}

Two phenomena dominate the loss of electrons from the X-Ray Ring: the Touschek effect and scattering from the residual gas. The Touschek effect describes the loss of particles from the ring when their energy deviation exceeds the energy acceptance. The Touschek half-life is proportional to the volume of the electron bunch ${ }^{[5]}$. Consequently, an emittance reduction tends to decrease the Touschek life-time. However, the energy acceptance of the ring is determined by both the size of the RF bucket and the dispersion of the lattice. The reduced dispersion and momentum compaction ( $50 \%$ lower) of the low emittance lattice both contribute to an increase in the energy acceptance and may counteract the reduction in the bunch volume.

Particles are lost to gas scattering when the transverse or longitudinal momentum imparted to an electron by an interaction with a residual gas molecule carries the electron outside of the transverse or momentum acceptance of the ring ${ }^{[6]}$. The reduced dispersion in the low emittance lattice will again tend to increase the gas scattering life-time while the larger beta function in the quadrupole triplet will tend to decrease it.

Clearly, the competing factors that influence the Touschek effect and residual gas scattering make the life-time of the low emittance lattice hard to predict but seem to counteract each other. The program ZAP ${ }^{[7]}$ was used to calculate the life-time from these effects for both the original and low emittance design lattices. The calculations used an RF overvoltage of 1.4 and did not take the synchrotron radiation losses in the insertion devices into account. The results of these calculations are plotted as a function of beam current in figure 4. Also shown are measured lifetimes in both lattices. The points for the original lattice represent data collected during six days in July and August, 1998. The low emittance results were obtained during five days in October, 1998. The ZAP results closely resemble the data. The life-time in the low emittance lattice has proven to be slightly greater but not appreciably different than in the original. 


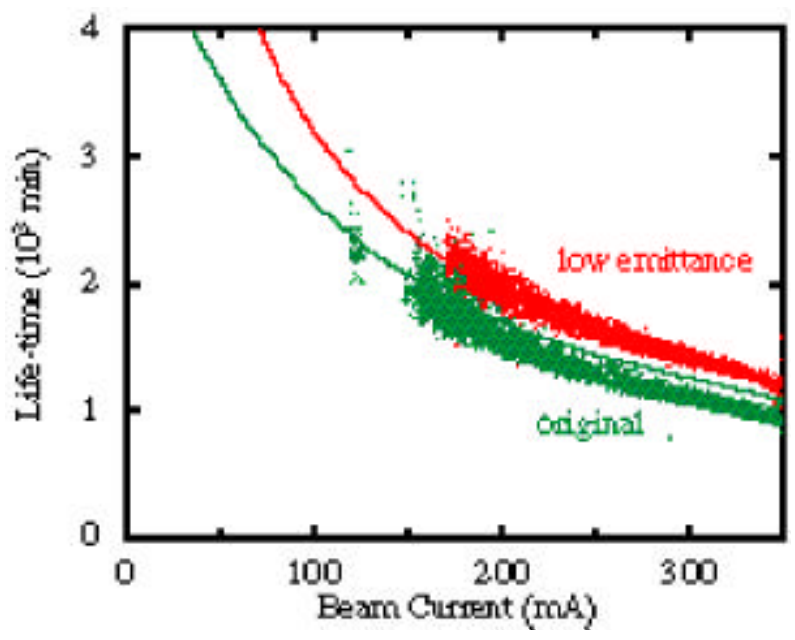

Figure 4: Measured and predicted life-times in the low emittance and original lattices.

\section{EFFECT ON USERS}

Most users are concerned only with the total flux of the synchrotron radiation and have not noticed any adverse effects from the low emittance lattice. The increase in life-time is an advantage but it is probably too small to notice. Certain users require tightly collimated x-ray beams and are more concerned with the brightness of the source than the flux. These users benefit greatly from the reduced emittance. Figure 5 compares energy spectra of the radiation produced by the $\mathrm{X} 1$ undulator in the forward direction. The two spectra were obtained from the low emittance and original lattices on successive $100 \mathrm{~mA}$ fills of the X-Ray Ring. The intensity of the first harmonic peak is approximately four times greater in the low emittance lattice.

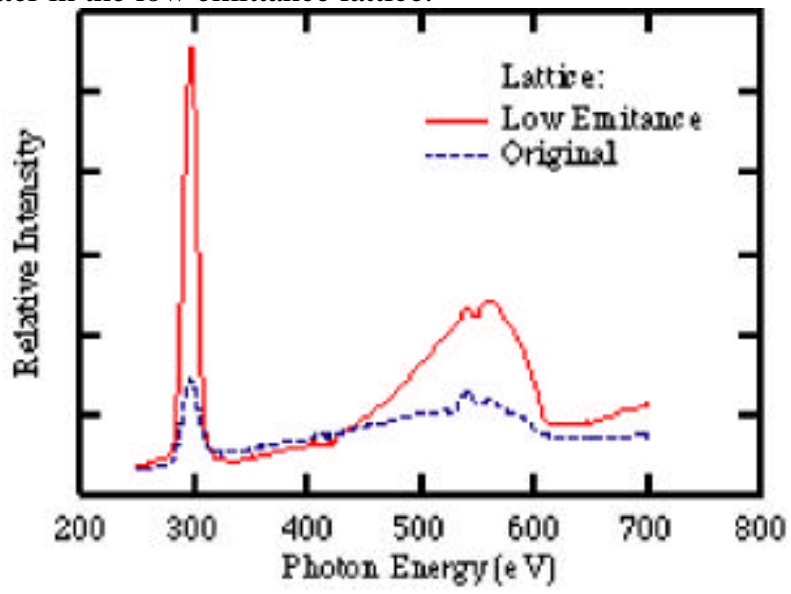

Figure 5: Photon energy spectra from the X1 undulator in the original and low emittance lattices. The beam current was $100 \mathrm{~mA}$ in both lattices.

\section{CONCLUSIONS}

The low emittance lattice has been successfully implemented in the NSLS X-Ray Ring at $2.584 \mathrm{GeV}$. The horizontal emittance was reduced to 46 from $90 \mathrm{~nm}$-rad with the vertical emittance maintained at $0.1 \mathrm{~nm}$-rad. Life-times are as good, or better than in the original lattice. The lattice will also be implemented at $2.8 \mathrm{GeV}$ when a new power supply and cabling are obtained to increase the current in sextupole magnets. 


\section{ACKNOWLEDGEMENTS}

The authors would like to thank Sue Wirick and Chris Jacobsen for measuring the radiation spectra shown in figure 5. They also thank Om Singh, Rich Biscardi, Yong Tang, Jim Murphy, and the rest of the NSLS staff for their help in implementing the low emittance lattice.

\section{REFERENCES}

${ }^{[1]}$ J. Safranek, "A Low Emittance Lattice for the NSLS X-Ray Ring", proceedings of the 1997 Particle Accelerator Conference.

${ }^{[2]}$ M. Sands, "The Physics of Electron Storage Rings: An Introduction", SLAC-121, Nov. 1970.

${ }^{[3]}$ J. Safranek, "Beam-Based Modeling and Control of Storage Rings", proceedings of the 1997 Particle Accelerator Conference.

${ }^{[4]}$ J. Safranek and S. Krinsky, "Plans to Increase Source Brightness of NSLS X-Ray Ring”, Proceedings of the 1993 Particle Accelerator Conference, 1491-1493.

${ }^{[5]}$ H. Bruck, Accelerateurs Circulaires de Particles, Presses Universitaires de France, Paris, 1966.

${ }^{[6]}$ J. LeDuff, "Current and Density Limitations in Existing Electron Storage Rings", Nucl. Instrum. Meth. A239, 1985, p. 83.

${ }^{[7]}$ M.S. Zisman, S. Chattopadhyay, and J.J. Bisognano, “Zap User’s Manual”, LBL-21270, 1986. 